\title{
An ambipolar host material provides highly efficient saturated red PhOLEDs possessing simple device structures $\dagger$
}

\author{
Wen-Yi Hung, ${ }^{* a}$ Tsung-Cheng Tsai, ${ }^{a}$ Sung-Yu Ku, ${ }^{b}$ Liang-Chen $\mathrm{Chi}^{b}$ and \\ Ken-Tsung Wong*b
}

Received 3rd July 2008, Accepted 25th July 2008

First published as an Advance Article on the web 13th August 2008

DOI: $10.1039 / \mathrm{b811356b}$

\begin{abstract}
A highly efficient red electrophosphorescent device exhibited saturated red emission and an impressive external quantum efficiency $\left[\eta_{\text {ext }}=10.8 \%(\mathrm{ph} / \mathrm{el})\right]$ with simple device configuration of doping an iridium complex ( $\mathrm{Mpq}_{2}$ Iracac) into a novel ambipolar spiro-configured donor-acceptor host material (D2ACN) has been developed.
\end{abstract}

Organic light-emitting diodes (OLEDs) are attracting much attention because of their potential applications in full-color flat-panel displays and lighting sources. ${ }^{1}$ Recently, particular interest has been aimed at phosphorescent OLEDs (PhOLEDs) because of their higher efficiencies that result from strong spin-orbit coupling effects induced by heavy metal centers. Transition metal-containing complexes serving as emitting guests, which are normally diluted into appropriate host materials, can harvest both singlet and triplet excitons for emission, providing the opportunity to realize internal quantum efficiencies close to $100 \%$ [external quantum efficiency $\left.\left(\eta_{\text {ext }}\right)=c a .20 \%(\mathrm{ph} / \mathrm{el})\right]^{2}$ To prevent reverse energy transfer in high-performance PhOLEDs, the host material must exhibit a triplet energy higher than that of the emitting phosphors. ${ }^{3}$ To balance electron and hole recombination within the emitting layer, sophisticated device configurations are usually required to facilitate efficient confinement of excitons on the phosphor. Unfortunately, such complicated and tedious fabrication processes would add considerably to the overall cost if used for the mass production of PhOLEDs. Therefore, the fabrication of small-molecule-based electroluminescent devices that combine high device performance with simple device configuration has become an attractive challenge. In this regard, the development of tailor-made molecules that fuse various functions into a multifunctional material is a potential alternative approach toward decreasing the complexity of device structures. Thus, materials possessing both hole- and electron-transporting (ambipolar) characteristics and good energy alignment with electrodes are potential candidates for use as active matrices for accommodating phosphors, thereby

\footnotetext{
${ }^{a}$ Institute of Optoelectronic Sciences, National Taiwan Ocean University, Keelung, Taiwan 202.

E-mail:wenhung@mail.ntou.edu.tw; Fax: +886224634360; Tel: + 886224622192 ext. 6718

${ }^{b}$ Department of Chemistry, National Taiwan University, Taipei,

Taiwan, 106.E-mail: kenwong@ntu.edu.tw; Fax: +8862 33661667; Tel: +886233661665

$\dagger$ Electronic supplementary information (ESI) available: Synthesis of $\mathrm{Mpq}_{2}$ Iracac, cyclic voltammogram, TOF mobilities measurements, and power efficiency of device. See DOI: 10.1039/b811356b
}

achieving highly efficient PhOLEDs having simple device configurations. Although a similar strategy has been employed previously for ambipolar materials been used as emitters in fluorescent OLEDs, ${ }^{4}$ very few ambipolar host materials have been incorporated successfully into PhOLEDs, ${ }^{5}$ presumably because of the arduous molecular design and synthesis of ambipolar host materials. Hence, the development of ambipolar host materials displaying balanced hole- and electrontransporting properties and suitable energy levels might result in a more cost-effective device fabrication process. In this Communication, we report a highly efficient red PhOLED possessing a simple device configuration and incorporating an Ir-complex doped into an ambipolar host. In the presence of a hole injection layer [mt-DATA:(4,4,4-tris(3-methylphenylphenylamino)triphenylamine)], this device exhibited saturated red emission (CIE: $x=0.66 ; y=0.34$ ) with high external quantum efficiency $(10.8 \%, \mathrm{ph} / \mathrm{el})$ and brightness $\left(23400 \mathrm{~cd} \mathrm{~m}^{-2}\right)$.

Fig. 1 displays the chemical structures of ambipolar host D2ACN and the Ir-centered dopant $\mathbf{M p q}_{2}$ Iracac. We have described the synthesis of D2ACN previously. ${ }^{6} \mathbf{D 2 A C N}$ is a spiro-configured donor (D)-acceptor (A) compound (i.e., a dual chromophore), in which electron-rich diphenylamino and electro-deficient cyano moieties are present on the two respective biphenyl branches of a 9,9'-spirobifluorene core. Fluorene derivatives presenting electron-donating arylamino groups are used widely as hole-transporting materials; ${ }^{7}$ the introduction of strong electron-accepting $\mathrm{CN}$ groups can enhance both the electron affinity ${ }^{8}$ for efficient electron injection from metal electrodes and electron transport. The fusion of these two functionalities allows D2ACN to function as a new ambipolar host material.

Differential scanning calorimetry (DSC) revealed that D2ACN possesses a glass transition temperature $\left(T_{\mathrm{g}}\right) \mathrm{ca}$. $116{ }^{\circ} \mathrm{C}$, suggesting that it could form homogeneous and

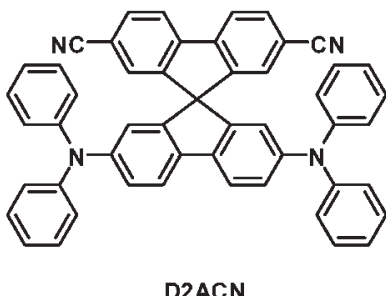

D2ACN

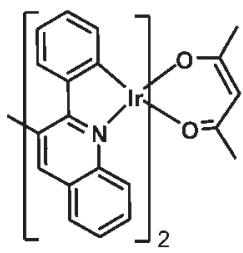

$\mathrm{Mpq}_{2}$ Iracac
Fig. 1 Chemical structures of $\mathbf{D 2 A C N}$ and $\mathrm{Mpq}_{2}$ Iracac. 
amorphous films through thermal evaporation. Thermogravimetric analysis (TGA) indicated that D2ACN possesses a high decomposition ( $10 \%$ weight loss) temperature $\left(T_{\mathrm{d}}\right)$ of $c a$. $350{ }^{\circ} \mathrm{C}$. We attribute the relatively high morphological and thermal stability of D2ACN to the perpendicular configuration of its spirobifluorene core, which tends to suppress crystallinity. ${ }^{9}$ In a study of the electrochemical behavior of D2ACN (Fig. S1, ESI $\dagger$ ), cyclic voltammetry (CV) revealed two reversible oxidation potentials at 0.79 and $1.17 \mathrm{~V}$ (vs. $\mathrm{Ag} / \mathrm{AgCl}$ ), which are slightly higher than those of 2,7-diphenylamino-9, $9^{\prime}$-spirobifluorene ${ }^{6}$ because of the presence of the electron poor $\mathrm{CN}$ groups; the large potential difference $(\Delta E=0.38 \mathrm{~V})$ indicates that the radical cation can be fully delocalized in the donor branch. We also detected one reversible reduction potential at $-1.61 \mathrm{~V}$. Accordingly, the $\mathrm{CV}$ results indicate that D2ACN exhibits bipolar character. From their redox potentials referenced to the redox couple of ferrocene, we estimated the HOMO and LUMO energy levels of D2ACN to be -5.14 and $-2.58 \mathrm{eV}$ respectively.

The photophysical property of D2ACN is shown in Fig. 2. D2ACN exhibits absorption peaks at 300, 330 and $383 \mathrm{~nm}$ that are a combination of the characteristics of the individual donor and acceptor chromophores, indicating the absence of ground-state electronic interactions between the donor and acceptor branches. Rather than emitting light from the two constituent chromophores individually, D2ACN is weakly fluorescent; we detect a weak and largely red-shifted yellow emission centered at $556 \mathrm{~nm}$, indicating that a significant photoinduced electron transfer occurred in excited state. ${ }^{6}$ We estimated the triplet energy of D2ACN to be $2.4 \mathrm{eV}$ $(\lambda=516 \mathrm{~nm})$ from the $0-0$ band of the low temperature (77 K) phosphorescence spectrum, rendering D2ACN as a host for a red phosphorescent guest.

We conducted charge-carrier mobility measurements for D2ACN at ambient temperature using a time-of-flight (TOF) technique. Both transients exhibit dispersive behavior, such that the carrier transit time $\left(t_{\mathrm{T}}\right)$ can be evaluated from the point of intersection of the two asymptotes in the double logarithmic representation (see Fig. S2, ESI $\dagger$ ). Using the equation $\mu=D^{2} V^{-1} t_{\mathrm{T}}{ }^{-1}$ ( $V$ : applied bias; $D$ : sample

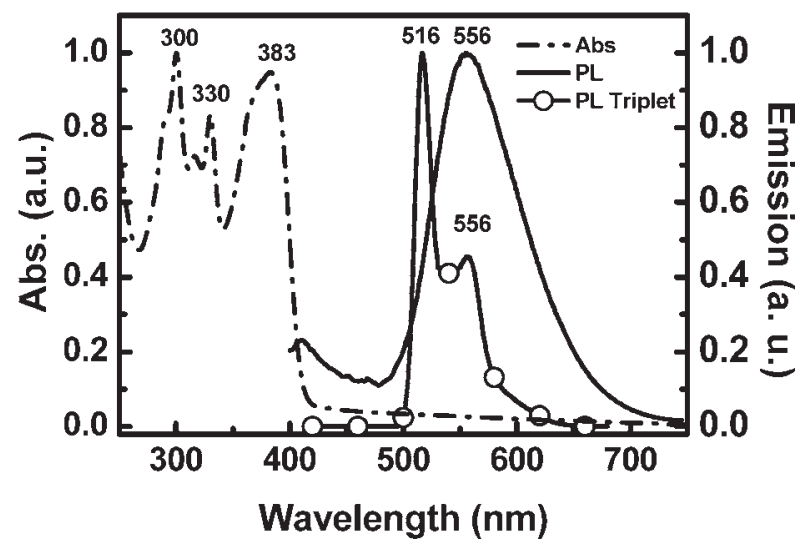

Fig. 2 Absorption and fluorescence spectra of a thin film of D2ACN (room temperature) and its phosphorescence spectrum in ethanol (77 K).

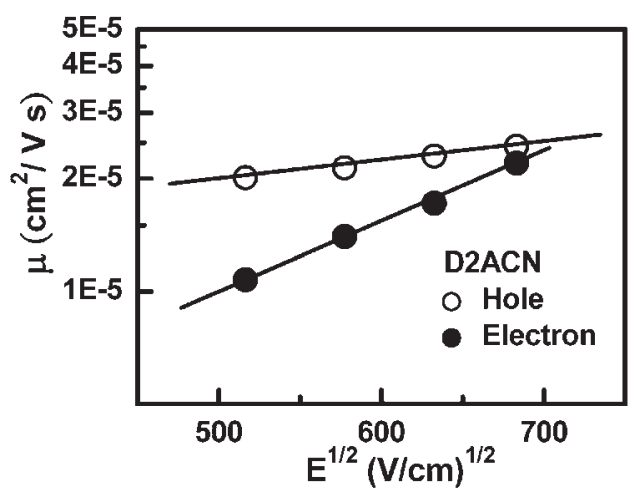

Fig. 3 Hole and electron mobilities plotted against $E^{1 / 2}$ for D2ACN.

thickness), we determined the hole and electron mobilities, which we display in Fig. 3 as a functions of the square root of the electric field $\left(E^{1 / 2}\right)$. The balanced bipolar charge transport capability of $\mathbf{D 2 A C N}$ is evidenced by the similar values (ca. $2 \times 10^{-5} \mathrm{~cm}^{2} \mathrm{~V}^{-1} \mathrm{~s}^{-1}$ ) of the electron and hole mobilities at $4.7 \times 10^{5} \mathrm{~V} \mathrm{~cm}^{-1}$. $^{10}$

Combining these observations together, we find that D2ACN possesses good thermal stability, balanced electron- and holetransporting ability, suitable HOMO/LUMO levels for charge injection, and an appropriate triplet energy gap for a red guest; thus, we expected it to serve as a host material for red PhOLEDs without the needing for incorporating charge transport layers. For device fabrication, we employed only a host/ guest layer and a hole-injection layer (HIL) in the following device configuration: ITO/mt-DATA $(20 \mathrm{~nm}) / \mathbf{D 2 A C N}$ : $\mathrm{Mpq}_{2} \mathrm{Iracac}(100 \mathrm{~nm}) / \mathrm{LiF}(0.5 \mathrm{~nm}) / \mathrm{Al}$. The addition of mt-DATA ${ }^{11}$ as the HIL between the ITO anode and the host/ guest layer is used widely to enhance hole injection and, hence, to improve the balance between the electron and hole currents. The use of other HILs (e.g. polyethylene dioxythiophene/polystyrene sulfonate, PEDOT:PSS), or their omission entirely, led the devices exhibiting lower efficiency and requiring higher operation potentials.

Optimizing the dopant concentration is an important step toward efficient Förster energy transfer. If the concentration is too low, energy transfer from the host to the guest will be incomplete because of the large average distance between the host and guest. ${ }^{12}$ If the concentration is too high, the concentration quenching effect (dipole-dipole deactivating interactions) will reduce the quantum efficiency of the device. To manifest the charge trapping effect of the dopants, we fabricated several devices having the same parameters, but with dopant concentrations ranging from 0.5 to $10 \mathrm{wt} \%$. We observed gradual increases in the external quantum efficiency $\left(\eta_{\text {ext }}\right)$ upon increasing the dopant concentration from 0.5 to $8 \mathrm{wt} \%$ in Fig. 4.

The power efficiency $\left(\eta_{\mathrm{p}}\right)$ was also observed the same condition (Fig. S3, ESI $\dagger$ ). The optimal performance was achieved at a doping concentration of $8 \mathrm{wt} \%$. The optimal values of $\eta_{\text {ext }}$ and $\eta_{\mathrm{p}}$ of $10.8 \%\left(\mathrm{ph} / \mathrm{el} ; 10.2 \mathrm{~cd} \mathrm{~A}^{-1}\right)$ and $13 \mathrm{~lm} \mathrm{~W}^{-1}$, respectively, occurred at a current density $(J)$ of $50 \mu \mathrm{A} \mathrm{cm}^{-2}$ and a luminance of $5 \mathrm{~cd} \mathrm{~m}^{-2}$. The device exhibited gradual decreases in the values of $\eta_{\text {ext }}$ and $\eta_{\mathrm{p}}$ upon increasing current density, presumably due to triplet-triplet annihilation. 


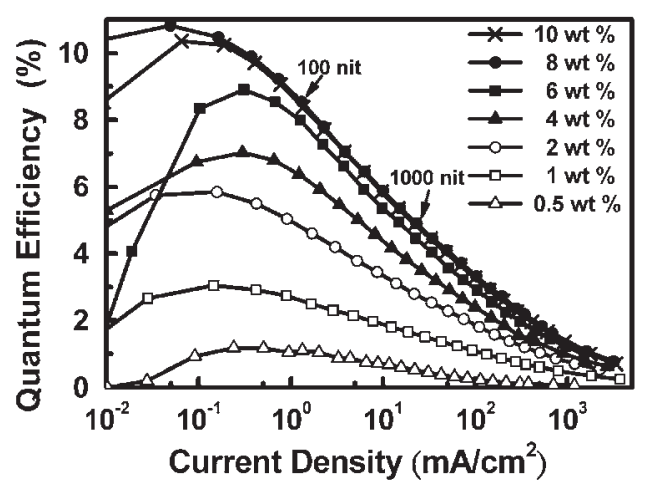

Fig. 4 External quantum efficiencies of devices incorporating various dopant concentrations, plotted as functions of the current density.

Even at a luminance of $1000 \mathrm{~cd} \mathrm{~m}^{-2}$, which corresponds to a value of $J$ of $23 \mathrm{~mA} \mathrm{~cm}^{-2}$, the device retained its high efficiency $\left[\eta_{\text {ext }}=4.9 \%(\mathrm{ph} / \mathrm{el}) ; \eta_{\mathrm{p}}=2 \mathrm{~lm} \mathrm{~W} \mathrm{~m}^{-1}\right]$. We also measured the absolute PL quantum efficiency $\left(\Phi_{\mathrm{PL}}\right)$ using a calibrated integration sphere (HAMAMATSU C9920). The value of $\Phi_{\mathrm{PL}}$ of the composite film (D2ACN: $8 \mathrm{wt} \% \mathrm{Mpg}_{2}$ Iracac) reached $c a$. $49 \%$, correlating theoretically to a device having an external quantum efficiency of $c a .10 \%(\mathrm{ph} / \mathrm{el})$, which is consistent with our observed device efficiency $\left[\eta_{\text {ext }}\right.$ $=10.8 \%(\mathrm{ph} / \mathrm{el})]$. This result clearly indicates that this device, with its simple structure, provided balanced charge recombination and good triplet exciton confinement within the phosphorescent dye.

Fig. 5 displays the $I-V-L$ characteristics of the devices incorporating dopant concentrations of 0.5 and $8 \mathrm{wt} \%$. Both devices exhibit rather low turn-on voltages of $1.5 \mathrm{~V}$ (defined as the voltage at which the EL is rapidly enhanced); subsequently, the current density and luminance increased upon increasing the doping concentration. The device doped with $8 \mathrm{wt} \%$ of the emitter achieved a maximum luminance of $23400 \mathrm{~cd} \mathrm{~m}^{-2}$, driven at a current density of $3200 \mathrm{~mA} \mathrm{~cm}{ }^{-2}$ $(13.5 \mathrm{~V})$. It is noteworthy, however, that the device retained a high luminance of $1000 \mathrm{~cd} \mathrm{~m}^{-2}$ at a current density of $1200 \mathrm{~mA} \mathrm{~cm}^{-2}(14 \mathrm{~V})$ even at the lower doping concentration $(0.5 \mathrm{wt} \%)$. These results imply that the guest molecules serve

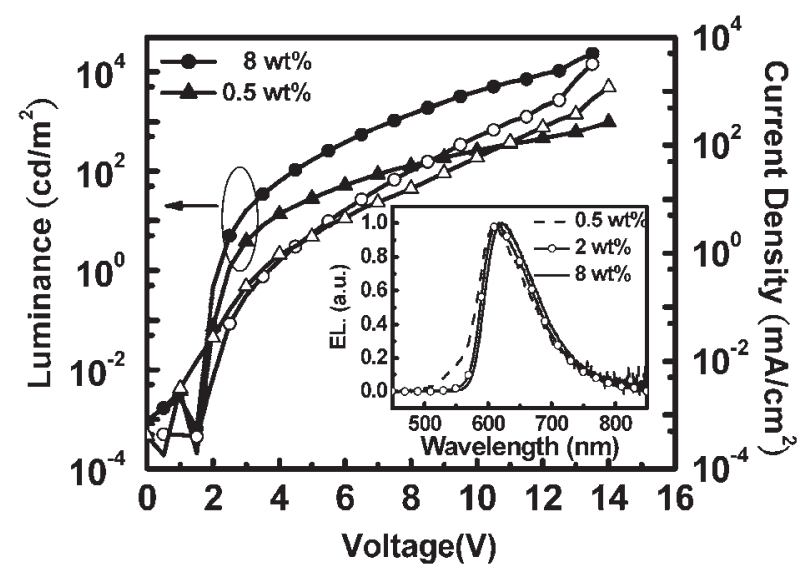

Fig. $5 I-V-L$ curves and EL spectra (inset) of devices incorporating various concentrations of the dopant. as charge trapping sites within the ambipolar host material D2ACN and account for the subsequent red emission, echoing our observation in Fig. 4 of the devices having much higher efficiencies at higher dopant concentrations. The inset to Fig. 5 displays the electroluminescence (EL) spectra of the devices containing three different dopant concentrations. When the concentration of $\mathrm{Mpq}_{2}$ Iracac was $0.5 \mathrm{wt} \%$, a weak emission appeared at $c a .550 \mathrm{~nm}$ together with a dominant emission peaking having a value of $\lambda_{\max }$ of $620 \mathrm{~nm}$. We attribute the high-energy emission to the photoinduced charge transfer emission of D2ACN. The EL emission from D2ACN was eliminated completely, however, when the dopant concentration reached $2 \mathrm{wt} \%$.

In conclusion, we reported a novel bipolar material, D2ACN, configured with appropriately positioned electrondeficient cyano and electron-rich diphenylamino moieties on the respective biphenyl branches of 9,9'-spirobifluorene, that possesses balanced ambipolar charge transport ability, suitable HOMO/LUMO levels, and a large triplet energy gap. These promising physical properties allow D2ACN to serve as an attractive host material for highly efficient red PhOLEDs. Because of the ambipolar character of D2ACN, the device structure can be simplified to ITO/mt-DATA/D2ACN: $\mathrm{Mpq}_{2} \mathrm{Iracac} / \mathrm{LiF} / \mathrm{Al}$. The optimized, highly efficient electrophosphorescent device incorporating $8 \mathrm{wt} \%$ of the red emitter exhibited a saturated red emission (CIE coordinates: $x=0.66$; $y=0.34$ ) having an impressive external quantum efficiency $\left[\eta_{\mathrm{ext}}=10.8 \%(\mathrm{ph} / \mathrm{el})\right]$ and a maximum power efficiency $\left(\eta_{\mathrm{p}}\right)$ of $13 \mathrm{~lm} \mathrm{~W}{ }^{-1}$. The ambipolar material acted as an effective charge conducting matrix to the host phosphor, which served as the charge trapping site for generating emissive excitons. Structural modification of D2ACN to enhance hole injection from the ITO electrode should facilitate the realization of a single-layer device (i.e. one that does not require a hole injection layer). These results of these ongoing investigations will be reported in due course.

The authors would like to acknowledge financial support from National Science Council of Taiwan.

\section{References}

1 C. W. Tang, S. A. VanSlyke and C. H. Chen, J. Appl. Phys., 1989, 65, 3610 .

2 M. A. Baldo, D. F. O'Brien, Y. You, A. Shoustikov, S. Sibley, M. E. Thompson and S. R. Forrest, Nature, 1998, 395, 151.

3 M. Sudhakar, P. I. Djurovich, T. E. Hogen-Esch and M. E. Thompson, J. Am. Chem. Soc., 2003, 125, 7796.

4 K. R. J. Thomas, J. T. Lin, M. Velusamy, Y.-T. Tao and C.-H. Chuen, Adv. Funct. Mater., 2004, 14, 83; C.-T. Chen, J.hyphen;S. Liu, M. V. R. K. Moturu, Y.-W. Lin, W. Yi, Y.-T. Tao and C.-H. Chen, Chem. Commun., 2005, 3980; Z. H. Li, M. S. Wong, H. Fukutani and Y. Tao, Org. Lett., 2006, 8, 4271; J. M. Hancock, A. P. Gifford, Y. Zhu, Y. Lou and S. A. Jenekhe, Chem. Mater., 2006, 18, 4924; Y.-L. Liao, C.-Y. Lin, K.-T. Wong, T.-H. Hou and W.-Y. Hung, Org. Lett., 2007, 9, 4511; T.-H. Huang, J. T. Lin, L.-Y. Chen, Y.-T. Lin and C.-C. Wu, Adv. Mater., 2006, 18, 602.

5 M.-Y. Lai, C.-H. Chen, W.-S. Huang, J. T. Lin, T.-H. Ke, L.-Y. Chen, M.-H. Tsai and C.-C. Wu, Angew. Chem., Int. Ed., 2008, 47, 581.

6 Y.-Y. Chien, K.-T. Wong, P.-T. Chou and Y.-M. Cheng, Chem. Commun., 2002, 2874; K.-T. Wong, S.-Y. Ku, Y.-M. Cheng, X.-Y. Lin, Y.-Y. Hung, S.-C. Pu, P.-T. Chou, G.-H. Lee and S.-M. Peng, J. Org. Chem., 2006, 71, 456.

7 Y. Shirota, J. Mater. Chem., 2000, 10, 1. 
8 X. Xu, S. Chen, G. Yu, C. Di, H. You, D. Ma and Y. Liu, $A d v$. Mater., 2007, 19, 1281.

9 T. P. I. Saragi, T. Spehr, A. Siebert, T. Fuhrmann-Lieker and J. Salbeck, Chem. Rev., 2007, 107, 1011.

10 Y.-L. Liao, C.-Y. Lin, K.-T. Wong, W.-Y. Hung and W.-J. Chen, Chem. Commun., 2007, 1831.
11 Y. Shirota, Y. Kuwabara, H. Inada, T. Wakimoto, H. Nakada, Y. Yonemoto, S. Kawami and K. Imai, Appl. Phys. Lett., 1994, 65, 807.

12 M. D. McGehee, T. Bergstedt, C. Zhang, A. P. Saab, M. B O'Regan, G. C. Bazan, V. I. Srdanov and A. J. Heeger, $A d v$. Mater., 1999, 11, 1349. 